Supplement of Earth Syst. Dynam., 8, 547-563, 2017

https://doi.org/10.5194/esd-8-547-2017-supplement

(C) Author(s) 2017. This work is distributed under

the Creative Commons Attribution 3.0 License.

(c) (1)
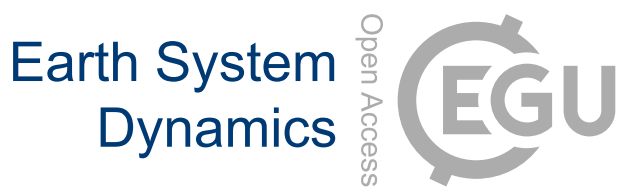

Supplement of

\title{
Emission metrics for quantifying regional climate impacts of aviation
}

Marianne T. Lund et al.

Correspondence to: Marianne T. Lund (m.t.lund@cicero.oslo.no)

The copyright of individual parts of the supplement might differ from the CC BY 3.0 License. 
5 Table S1: Global and regional aviation emissions used in this study. Emissions are for year 20066 and from the AEDT inventory (Wilkerson et al., 2010). Also included is the accumulated flight 7 distance in each region.

\begin{tabular}{l|cccccc}
\hline $\begin{array}{l}\text { Source } \\
\text { Region }\end{array}$ & $\begin{array}{c}\mathrm{BC} \\
\left(\mathrm{kg} \mathrm{yr}^{-1}\right)\end{array}$ & $\begin{array}{c}\mathrm{NOx} \\
(\mathrm{kgN} \mathrm{yr}-1)\end{array}$ & $\begin{array}{c}\mathrm{OC} \\
\left(\mathrm{kg} \mathrm{yr}^{-1}\right)\end{array}$ & $\begin{array}{c}\mathrm{SO} 2 \\
\left(\mathrm{~kg} \mathrm{yr}^{-1}\right)\end{array}$ & $\begin{array}{c}\mathrm{CO} 2 \\
\left(\mathrm{~kg} \mathrm{yr}^{-1}\right)\end{array}$ & $\begin{array}{c}\text { Flight distance } \\
(\mathrm{km})\end{array}$ \\
\hline Global & $5.9 \mathrm{E}+06$ & $8.1 \mathrm{E}+08$ & $6.4 \mathrm{E}+06$ & $2.3 \mathrm{E}+08$ & $5.9 \mathrm{E}+11$ & $6.7 \mathrm{E}+10$ \\
SAF & $4.0 \mathrm{E}+05$ & $5.6 \mathrm{E}+07$ & $3.6 \mathrm{E}+05$ & $1.4 \mathrm{E}+07$ & $3.8 \mathrm{E}+10$ & $2.5 \mathrm{E}+09$ \\
NAM & $2.2 \mathrm{E}+06$ & $2.9 \mathrm{E}+08$ & $2.0 \mathrm{E}+06$ & $8.2 \mathrm{E}+07$ & $2.2 \mathrm{E}+11$ & $1.8 \mathrm{E}+10$ \\
EAS & $9.8 \mathrm{E}+05$ & $1.6 \mathrm{E}+08$ & $1.1 \mathrm{E}+06$ & $3.8 \mathrm{E}+07$ & $1.0 \mathrm{E}+11$ & $4.6 \mathrm{E}+09$ \\
EUR & $1.5 \mathrm{E}+06$ & $2.2 \mathrm{E}+08$ & $2.1 \mathrm{E}+06$ & $5.8 \mathrm{E}+07$ & $1.5 \mathrm{E}+11$ & $8.9 \mathrm{E}+09$ \\
SPO & $4.6 \mathrm{E}+05$ & $7.1 \mathrm{E}+07$ & $4.8 \mathrm{E}+05$ & $1.8 \mathrm{E}+07$ & $4.6 \mathrm{E}+10$ & $2.4 \mathrm{E}+09$ \\
SAS & $2.5 \mathrm{E}+05$ & $4.1 \mathrm{E}+07$ & $2.3 \mathrm{E}+05$ & $9.5 \mathrm{E}+06$ & $2.5 \mathrm{E}+10$ & $1.6 \mathrm{E}+09$ \\
\hline
\end{tabular}

8

9

10 Table S2: Radiative forcing $\left(\mathrm{Wm}^{-2}\right)$ by component, source region and latitude band for input to

11 the emission metric calculations.

\begin{tabular}{ll|ccccc}
\hline \multirow{2}{*}{ Component } & \multicolumn{5}{|c}{$\begin{array}{c}\text { Radiative forcing } \\
\left(\mathrm{Wm}^{-2}\right)\end{array}$} \\
\hline Contrail- & Source region & Global & $90^{\circ} \mathrm{S}-28^{\circ} \mathrm{S}$ & $28^{\circ} \mathrm{S}-28^{\circ} \mathrm{N}$ & $28^{\circ} \mathrm{N}-60^{\circ} \mathrm{N}$ & $60^{\circ} \mathrm{N}-90^{\circ} \mathrm{N}$ \\
Cirrus & Global & $4.6 \mathrm{E}-02$ & $1.8 \mathrm{E}-03$ & $2.8 \mathrm{E}-02$ & $1.6 \mathrm{E}-01$ & $2.1 \mathrm{E}-02$ \\
& SAF & $3.5 \mathrm{E}-03$ & $4.7 \mathrm{E}-04$ & $6.5 \mathrm{E}-03$ & $9.7 \mathrm{E}-05$ & $9.0 \mathrm{E}-10$ \\
& NAM & $1.8 \mathrm{E}-02$ & $-1.5 \mathrm{E}-10$ & $7.8 \mathrm{E}-03$ & $7.5 \mathrm{E}-02$ & $5.5 \mathrm{E}-03$ \\
& EAS & $4.2 \mathrm{E}-03$ & $-4.8 \mathrm{E}-10$ & $4.3 \mathrm{E}-03$ & $1.0 \mathrm{E}-02$ & $1.2 \mathrm{E}-03$ \\
& EUR & $9.5 \mathrm{E}-03$ & $2.0 \mathrm{E}-10$ & $1.2 \mathrm{E}-04$ & $4.9 \mathrm{E}-02$ & $7.0 \mathrm{E}-03$ \\
& SPO & $2.7 \mathrm{E}-03$ & $7.3 \mathrm{E}-04$ & $4.9 \mathrm{E}-03$ & $-1.0 \mathrm{E}-06$ & $2.5 \mathrm{E}-11$ \\
& SAS & $1.6 \mathrm{E}-03$ & $-2.0 \mathrm{E}-10$ & $3.0 \mathrm{E}-03$ & $6.1 \mathrm{E}-04$ & $9.3 \mathrm{E}-09$ \\
\hline \multirow{2}{*}{ NOx-ozone } & $1.9 \mathrm{E}-02$ & $4.8 \mathrm{E}-03$ & $2.0 \mathrm{E}-02$ & $3.6 \mathrm{E}-02$ & $2.3 \mathrm{E}-02$ \\
& Global & $2.0 \mathrm{E}-03$ & $1.4 \mathrm{E}-03$ & $2.9 \mathrm{E}-03$ & $1.0 \mathrm{E}-03$ & $2.5 \mathrm{E}-04$ \\
& SAF & $5.3 \mathrm{E}-03$ & $7.0 \mathrm{E}-05$ & $4.0 \mathrm{E}-03$ & $1.4 \mathrm{E}-02$ & $8.4 \mathrm{E}-03$ \\
& NAM & $4.0 \mathrm{E}-03$ & $3.6 \mathrm{E}-04$ & $4.8 \mathrm{E}-03$ & $6.9 \mathrm{E}-03$ & $3.8 \mathrm{E}-03$ \\
& EAS & $3.0 \mathrm{E}-03$ & $4.7 \mathrm{E}-05$ & $1.3 \mathrm{E}-03$ & $9.0 \mathrm{E}-03$ & $8.2 \mathrm{E}-03$ \\
& EUR & $3.1 \mathrm{E}-03$ & $2.5 \mathrm{E}-03$ & $4.8 \mathrm{E}-03$ & $7.8 \mathrm{E}-04$ & $-5.1 \mathrm{E}-05$
\end{tabular}




\begin{tabular}{|c|c|c|c|c|c|c|}
\hline & SAS & $1.6 \mathrm{E}-03$ & 7.0E-05 & $2.2 \mathrm{E}-03$ & $2.4 \mathrm{E}-03$ & $6.6 \mathrm{E}-04$ \\
\hline \multirow[t]{7}{*}{$B C$} & Global & $5.7 \mathrm{E}-04$ & $9.7 \mathrm{E}-05$ & $5.2 \mathrm{E}-04$ & $1.3 \mathrm{E}-03$ & $6.6 \mathrm{E}-04$ \\
\hline & SAF & $5.4 \mathrm{E}-05$ & $2.8 \mathrm{E}-05$ & $8.2 \mathrm{E}-05$ & $2.6 \mathrm{E}-05$ & $9.9 \mathrm{E}-06$ \\
\hline & NAM & $1.9 \mathrm{E}-04$ & $1.8 \mathrm{E}-06$ & $1.3 \mathrm{E}-04$ & $5.9 \mathrm{E}-04$ & 2.7E-04 \\
\hline & EAS & $1.0 \mathrm{E}-04$ & $9.0 \mathrm{E}-06$ & $1.1 \mathrm{E}-04$ & $2.0 \mathrm{E}-04$ & $1.0 \mathrm{E}-04$ \\
\hline & EUR & $1.1 \mathrm{E}-04$ & $1.4 \mathrm{E}-06$ & 4.7E-05 & 4.0E-04 & $2.2 \mathrm{E}-04$ \\
\hline & SPO & $5.6 \mathrm{E}-05$ & 4.9E-05 & $8.5 E-05$ & $1.3 \mathrm{E}-05$ & 7.3E-06 \\
\hline & SAS & $5.0 \mathrm{E}-05$ & $3.8 \mathrm{E}-06$ & $6.4 \mathrm{E}-05$ & 7.7E-05 & $3.3 \mathrm{E}-05$ \\
\hline \multirow[t]{7}{*}{ OC } & Global & $-4.6 \mathrm{E}-05$ & $-4.7 E-06$ & $-4.9 \mathrm{E}-05$ & $-9.9 E-05$ & $-2.1 \mathrm{E}-05$ \\
\hline & SAF & $-4.4 \mathrm{E}-06$ & $-1.4 \mathrm{E}-06$ & $-7.3 \mathrm{E}-06$ & $-1.6 \mathrm{E}-06$ & $-2.5 \mathrm{E}-07$ \\
\hline & NAM & $-1.5 \mathrm{E}-05$ & $-6.4 \mathrm{E}-08$ & $-1.3 \mathrm{E}-05$ & $-4.1 \mathrm{E}-05$ & $-7.8 \mathrm{E}-06$ \\
\hline & EAS & $-7.7 E-06$ & $-4.0 \mathrm{E}-07$ & $-1.0 \mathrm{E}-05$ & $-1.2 \mathrm{E}-05$ & $-2.9 \mathrm{E}-06$ \\
\hline & EUR & $-1.0 \mathrm{E}-05$ & $-5.3 E-08$ & $-5.3 E-06$ & $-3.8 \mathrm{E}-05$ & $-8.8 \mathrm{E}-06$ \\
\hline & SPO & $-5.1 E-06$ & $-2.5 \mathrm{E}-06$ & $-8.5 E-06$ & $-8.7 E-07$ & $-2.1 \mathrm{E}-07$ \\
\hline & SAS & $-3.6 \mathrm{E}-06$ & $-1.4 \mathrm{E}-07$ & $-5.4 \mathrm{E}-06$ & $-4.2 \mathrm{E}-06$ & $-8.3 \mathrm{E}-07$ \\
\hline \multirow[t]{7}{*}{ Sulfate } & Global & $-3.2 E-03$ & $-2.2 \mathrm{E}-04$ & $-3.5 E-03$ & $-6.5 E-03$ & $-2.0 \mathrm{E}-03$ \\
\hline & SAF & $-3.0 \mathrm{E}-04$ & $-9.9 E-05$ & $-5.0 \mathrm{E}-04$ & $-1.1 \mathrm{E}-04$ & $-1.8 \mathrm{E}-05$ \\
\hline & NAM & $-1.1 \mathrm{E}-03$ & 3.6E-06 & $-1.0 \mathrm{E}-03$ & $-3.0 \mathrm{E}-03$ & $-8.5 \mathrm{E}-04$ \\
\hline & EAS & $-5.8 \mathrm{E}-04$ & $-3.2 \mathrm{E}-06$ & $-7.5 \mathrm{E}-04$ & $-9.7 E-04$ & $-2.7 \mathrm{E}-04$ \\
\hline & EUR & $-5.5 E-04$ & 1.0E-06 & $-2.9 \mathrm{E}-04$ & $-1.9 \mathrm{E}-03$ & $-7.8 \mathrm{E}-04$ \\
\hline & SPO & $-3.3 E-04$ & $-1.1 \mathrm{E}-04$ & $-5.8 \mathrm{E}-04$ & $-4.1 \mathrm{E}-05$ & $7.2 \mathrm{E}-06$ \\
\hline & SAS & $-2.7 \mathrm{E}-04$ & $-2.9 \mathrm{E}-06$ & $-3.8 \mathrm{E}-04$ & $-3.5 E-04$ & $-7.8 \mathrm{E}-05$ \\
\hline \multirow[t]{7}{*}{ NOx-nitrate } & Global & $-3.2 \mathrm{E}-04$ & $-5.6 \mathrm{E}-07$ & $-2.8 \mathrm{E}-04$ & $-9.7 E-04$ & $-4.1 \mathrm{E}-05$ \\
\hline & SAF & $-1.2 \mathrm{E}-05$ & $-1.9 \mathrm{E}-07$ & $-1.7 E-05$ & $-1.9 \mathrm{E}-05$ & $-4.8 \mathrm{E}-08$ \\
\hline & NAM & $-9.4 \mathrm{E}-05$ & $5.8 \mathrm{E}-10$ & $-8.0 \mathrm{E}-05$ & $-2.8 \mathrm{E}-04$ & $-1.3 \mathrm{E}-05$ \\
\hline & EAS & $-3.2 \mathrm{E}-05$ & 6.0E-09 & $-2.4 \mathrm{E}-05$ & $-1.1 \mathrm{E}-04$ & $-6.1 \mathrm{E}-06$ \\
\hline & EUR & $-1.1 \mathrm{E}-04$ & $-3.9 \mathrm{E}-09$ & $-6.7 E-05$ & $-4.2 \mathrm{E}-04$ & $-1.9 \mathrm{E}-05$ \\
\hline & SPO & $-5.9 E-06$ & $-3.5 \mathrm{E}-07$ & $-8.3 E-06$ & $-8.5 E-06$ & $-1.8 \mathrm{E}-08$ \\
\hline & SAS & $-4.1 \mathrm{E}-05$ & 1.7E-09 & $-5.7 E-05$ & $-6.4 \mathrm{E}-05$ & $-3.2 \mathrm{E}-07$ \\
\hline \multirow[t]{7}{*}{ NOx-methane } & Global & $-9.3 E-03$ & $-6.8 \mathrm{E}-03$ & $-1.1 \mathrm{E}-02$ & $-8.6 \mathrm{E}-03$ & $-6.1 \mathrm{E}-03$ \\
\hline & SAF & $-1.1 \mathrm{E}-03$ & $-8.3 \mathrm{E}-04$ & $-1.4 \mathrm{E}-03$ & $-1.0 \mathrm{E}-03$ & $-7.4 \mathrm{E}-04$ \\
\hline & NAM & $-2.7 \mathrm{E}-03$ & $-2.0 \mathrm{E}-03$ & $-3.3 E-03$ & $-2.5 E-03$ & $-1.8 \mathrm{E}-03$ \\
\hline & EAS & $-1.7 \mathrm{E}-03$ & $-1.2 \mathrm{E}-03$ & $-2.0 \mathrm{E}-03$ & $-1.5 \mathrm{E}-03$ & $-1.1 \mathrm{E}-03$ \\
\hline & EUR & $-1.5 \mathrm{E}-03$ & $-1.1 \mathrm{E}-03$ & $-1.8 \mathrm{E}-03$ & $-1.4 \mathrm{E}-03$ & $-9.7 E-04$ \\
\hline & SPO & $-1.4 \mathrm{E}-03$ & $-9.0 \mathrm{E}-04$ & $-1.5 \mathrm{E}-03$ & $-1.1 \mathrm{E}-03$ & $-8.1 E-04$ \\
\hline & SAS & $-7.0 \mathrm{E}-04$ & $-4.5 E-04$ & $-7.4 \mathrm{E}-04$ & $-5.6 E-04$ & $-4.0 \mathrm{E}-04$ \\
\hline
\end{tabular}

12 
17 Table S3: Regional climate sensitivities (RCS) used in the emission metric calculations [K (W $\left.\left.18 \mathrm{~m}^{-2}\right)^{-1}\right]$ (Shindell \& Faluvegi, 2009).

\begin{tabular}{|c|c|c|c|c|c|}
\hline \multirow{2}{*}{$\begin{array}{l}\text { Sulfate, OC, nitrate, } \\
\text { methane, contrail-cirrus }\end{array}$} & \multicolumn{5}{|c|}{ Forcing region } \\
\hline & & $90^{\circ} \mathrm{S}-28^{\circ} \mathrm{S}$ & $28^{\circ} \mathrm{S}-28^{\circ} \mathrm{N}$ & $28^{\circ} \mathrm{N}-60^{\circ} \mathrm{N}$ & $60^{\circ} \mathrm{N}-90^{\circ} \mathrm{N}$ \\
\hline \multirow{4}{*}{ Response region } & $90^{\circ} \mathrm{S}-28^{\circ} \mathrm{S}$ & 0.19 & 0.05 & 0.02 & 0 \\
\hline & $28^{\circ} \mathrm{S}-28^{\circ} \mathrm{N}$ & 0.09 & 0.24 & 0.1 & 0.02 \\
\hline & $28^{\circ} \mathrm{N}-60^{\circ} \mathrm{N}$ & 0.07 & 0.17 & 0.24 & 0.06 \\
\hline & $60^{\circ} \mathrm{N}-90^{\circ} \mathrm{N}$ & 0.06 & 0.16 & 0.17 & 0.31 \\
\hline \multirow{2}{*}{ NOx-induced ozone change } & \multicolumn{5}{|c|}{ Forcing region } \\
\hline & & $90^{\circ} \mathrm{S}-28^{\circ} \mathrm{S}$ & $28^{\circ} \mathrm{S}-28^{\circ} \mathrm{N}$ & $28^{\circ} \mathrm{N}-60^{\circ} \mathrm{N}$ & $60^{\circ} \mathrm{N}-90^{\circ} \mathrm{N}$ \\
\hline \multirow{4}{*}{ Response region } & $90^{\circ} \mathrm{S}-28^{\circ} \mathrm{S}$ & 0.19 & 0.13 & -0.06 & -0.03 \\
\hline & $28^{\circ} \mathrm{S}-28^{\circ} \mathrm{N}$ & 0.09 & 0.26 & 0.09 & 0.02 \\
\hline & $28^{\circ} \mathrm{N}-60^{\circ} \mathrm{N}$ & 0.07 & 0.15 & 0.2 & 0.06 \\
\hline & $60^{\circ} \mathrm{N}-90^{\circ} \mathrm{N}$ & 0.06 & 0.13 & 0.05 & 0.07 \\
\hline \multirow{2}{*}{ BC } & \multicolumn{5}{|c|}{ Forcing region } \\
\hline & & $90^{\circ} \mathrm{S}-28^{\circ} \mathrm{S}$ & $28^{\circ} \mathrm{S}-28^{\circ} \mathrm{N}$ & $28^{\circ} \mathrm{N}-60^{\circ} \mathrm{N}$ & $60^{\circ} \mathrm{N}-90^{\circ} \mathrm{N}$ \\
\hline \multirow{4}{*}{ Response region } & $90^{\circ} \mathrm{S}-28^{\circ} \mathrm{S}$ & 0.19 & 0.06 & 0.02 & 0 \\
\hline & $28^{\circ} \mathrm{S}-28^{\circ} \mathrm{N}$ & 0.09 & 0.17 & 0.07 & 0.02 \\
\hline & $28^{\circ} \mathrm{N}-60^{\circ} \mathrm{N}$ & 0.07 & 0.24 & 0.14 & 0.08 \\
\hline & $60^{\circ} \mathrm{N}-90^{\circ} \mathrm{N}$ & 0.06 & 0.31 & 0.15 & -0.08 \\
\hline
\end{tabular}

19

20

21

22

23

24

25

26

27

28

29

Table S4 Relative uncertainties adopted in the Monte Carlo analysis.

\begin{tabular}{|l|l|l|}
\hline & $\begin{array}{l}\text { Relative } \\
\text { uncertainty (1 SD) }\end{array}$ & Source \\
\hline $\mathrm{BC}$ & $39 \%$ & AeroCom multi-model mean (Myhre et al., 2013a) \\
\hline $\mathrm{SO} 2$ (sulfate) & $34 \%$ & AeroCom multi-model mean (Myhre et al. 2013a) \\
\hline $\mathrm{OC}$ & $33 \%$ & AeroCom multi-model mean (Myhre et al. 2013a) \\
\hline $\mathrm{NOx}$ (nitrate) & $50 \%$ & AeroCom multi-model mean (Myhre et al. 2013a) \\
\hline $\mathrm{NOx}$ & $73 \%$ & IPCC AR5, WG1 Ch.8 SM (Myhre et al., 2013b) \\
\hline $\mathrm{CO} 2$ & $6 \%$ & IPCC AR5, WG1 Ch.8 SM (Myhre et al. 2013b) \\
\hline
\end{tabular}

(1)

1


30 Table S5: Global and regional GWP and GTP of contrail-cirrus for time horizons 20 and 100 31 years, calculated per $\mathrm{km}$ accumulated flight distance. The GTPs are calculated using the impulse 32 response function by Boucher and Reddy (2008) and values are given relative to $\mathrm{CO}_{2}$ using $\mathrm{CO}_{2}$ 33 parameters from Joos et al. (2013).

\begin{tabular}{ll|cc|cc}
\hline & & \multicolumn{2}{|c|}{ GWP } & \multicolumn{2}{c}{ GTP } \\
\hline Component & $\begin{array}{l}\text { Source } \\
\text { region }\end{array}$ & $\mathrm{H}=20$ & $\mathrm{H}=100$ & $\mathrm{H}=20$ & $\mathrm{H}=100$ \\
\hline Contrail- & Global & 27 & 7.5 & 8 & 1.1 \\
cirrus & SAF & 55 & 15 & 17 & 2.2 \\
& NAM & 41 & 11 & 12 & 1.6 \\
& EAS & 36 & 10 & 11 & 1.4 \\
& EUR & 42 & 11 & 13 & 1.6 \\
& SPO & 44 & 12 & 13 & 1.7 \\
& SAS & 40 & 11 & 12 & 1.6 \\
\hline
\end{tabular}

34

35

36 

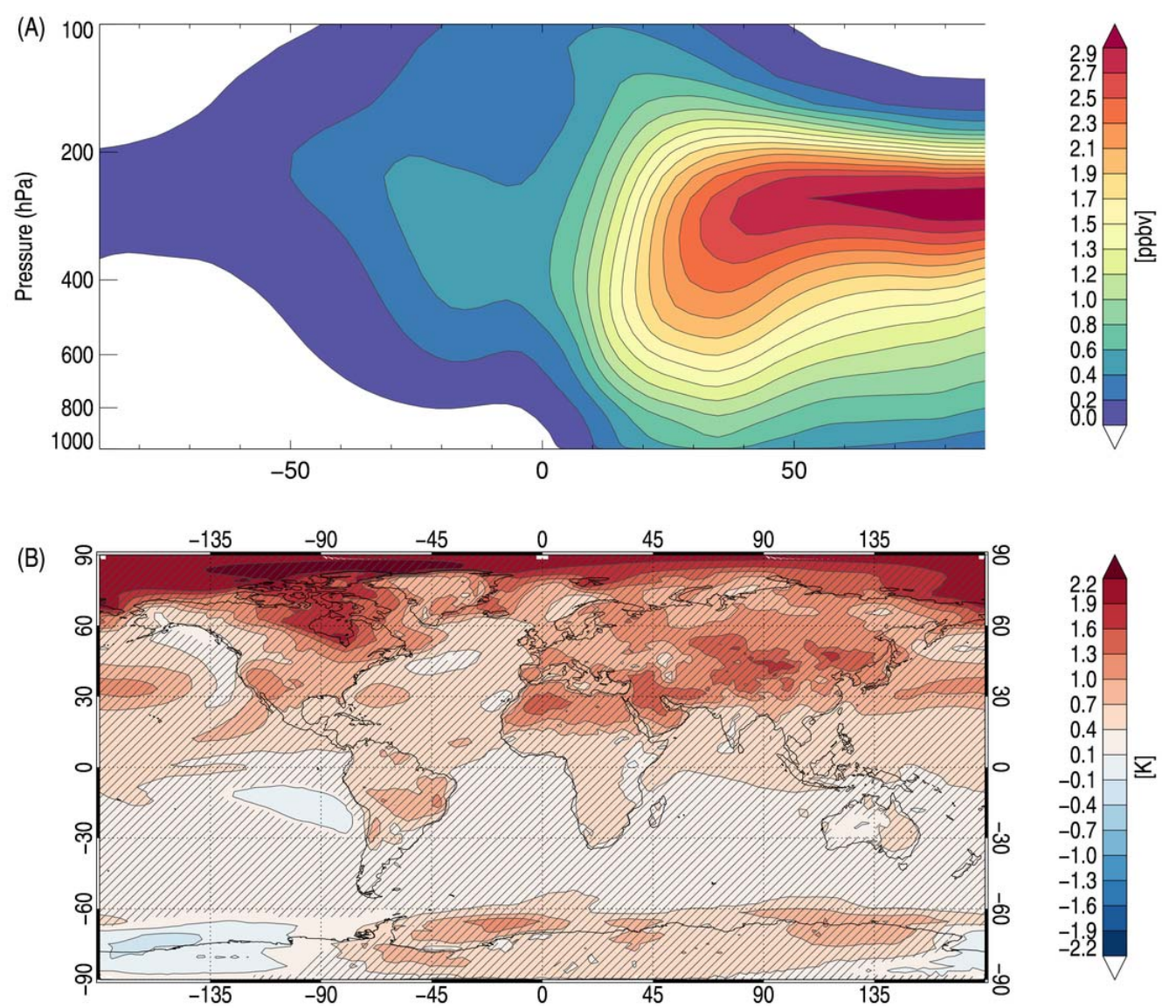

38 Figure S1: (A) Zonal, annual mean ozone concentration change from OsloCTM3 caused by 39 global aviation NOx emissions and (B) annual mean surface temperature response to the aviation 40 ozone perturbation (scaled by a factor 40) as simulated by CESM1.2. Hatching indicates statistical 41 significance at the 0.05 level.

42 


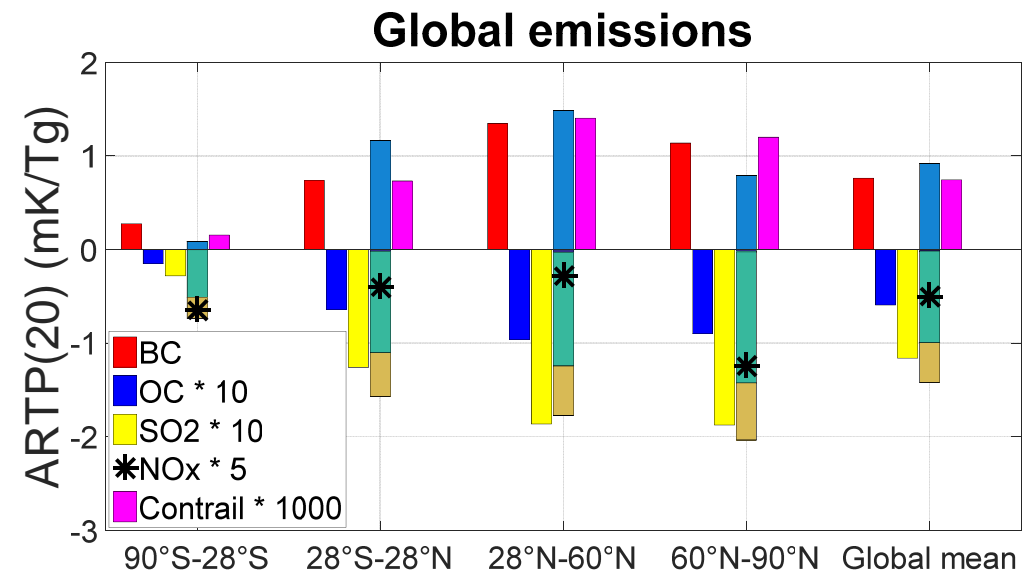

49 Figure 2: $\operatorname{ARTP(20)}$ for $\mathrm{BC}, \mathrm{OC}, \mathrm{SO}_{2}, \mathrm{NOx}$ and contrail-cirrus for global aviation emissions.

50 NOx is comprised of contributions from ozone (light blue), methane (teal green), methane-induced

51 ozone (dark yellow) changes and nitrate aerosols (dark blue). The asterisk indicate the net

NOx 52 effect.

53

54

55

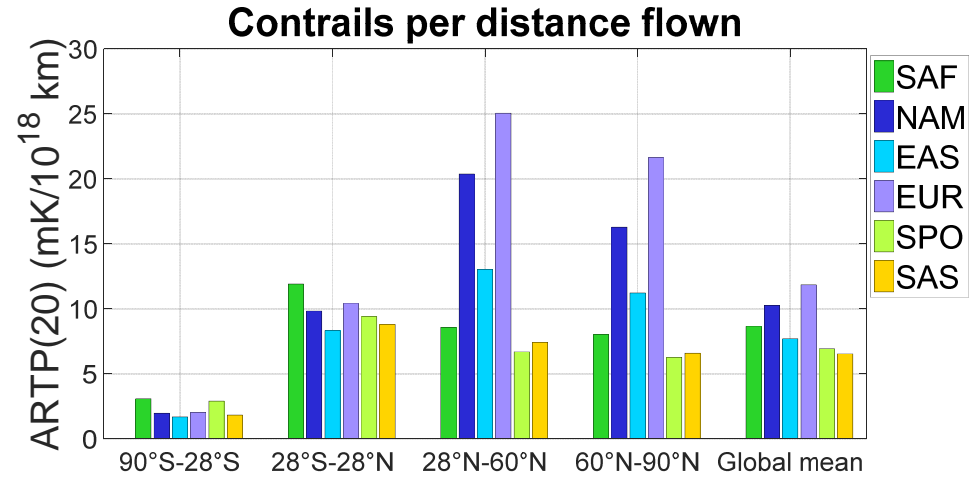

56 Figure S3: ARTP(20) of aviation-induced contrail-cirrus calculated per accumulated km flight

57 distance.

58

59

60

61 


\section{References}

Joos F., Roth R., Fuglestvedt J. S., Peters G. P., Enting I. G., von Bloh W., Brovkin V., Burke E. J., Eby M., Edwards N. R., Friedrich T., Frölicher T. L., Halloran P. R., Holden P. B., Jones C., Kleinen T., Mackenzie F. T., Matsumoto K., Meinshausen M., Plattner G. K., Reisinger A., Segschneider J., Shaffer G., Steinacher M., Strassmann K., Tanaka K., Timmermann A. \& Weaver A. J. (2013). Carbon dioxide and climate impulse response functions for the computation of greenhouse gas metrics: a multi-model analysis. Atmos. Chem. Phys. 13(5), 2793-2825, DOI: 10.5194/acp-13-2793-2013.

Myhre G., Samset B. H., Schulz M., Balkanski Y., Bauer S., Berntsen T. K., Bian H., Bellouin N., Chin M., Diehl T., Easter R. C., Feichter J., Ghan S. J., Hauglustaine D., Iversen T., Kinne S., Kirkevåg A., Lamarque J. F., Lin G., Liu X., Lund M. T., Luo G., Ma X., van Noije T., Penner J. E., Rasch P. J., Ruiz A., Seland $\varnothing$., Skeie R. B., Stier P., Takemura T., Tsigaridis K., Wang P., Wang Z., Xu L., Yu H., Yu F., Yoon J. H., Zhang K., Zhang H. \& Zhou C. (2013a). Radiative forcing of the direct aerosol effect from AeroCom Phase II simulations. Atmos. Chem. Phys. 13(4), 1853-1877, DOI: 10.5194/acp-13-1853-2013.

Myhre G., Shindell D., Brèon F.-M., Collins W., Fuglestvedt J., Huang J., Koch D., Lamarque J.-F., Lee D., Mendoza B., Nakajima T., Robock A., Stephens G., Takemura T. \& Zhang H. (2013b). Anthropogenic and natural radiative forcing. In: Climate Change 2013: The Physical Science Basis. Contribution of Working Group I to the Fifth Assessment Report of the Intergovernmental Panel on Climate Change [Stocker, T.F., D., Qin, G.-K. Plattner, M. Tignor, S.K. Allen, J. Boschung, A. Nauels, Y. Xia, V. Bex and P.M. Midgley (eds). Cambridge University Press, Cambridge, United Kingdom and New York, NY, USA

Shindell D. \& Faluvegi G. (2009). Climate response to regional radiative forcing during the twentieth century. Nature Geoscience. 2(4), 294-300, DOI: 10.1038/ngeo473.

Wilkerson J. T., Jacobson M. Z., Malwitz A., Balasubramanian S., Wayson R., Fleming G., Naiman A. D. \& Lele S. K. (2010). Analysis of emission data from global commercial aviation: 2004 and 2006. Atmos. Chem. Phys. 10(13), 6391-6408, DOI: 10.5194/acp-10-6391-2010. 